\title{
Prematurity-related hypertension in children and adolescents born at the Baruch-Padeh medical center, Poriya, North Israel
}

\author{
Aluma Elkayam ${ }^{1}$, Ahmed Abuleil ${ }^{1,2}$, Smmolken T ${ }^{3}$, Az Saed ${ }^{2}$, Haia N ${ }^{1}$, Boshra Nasser $^{1}$ and Wael Nasser $^{1,2 *}$ \\ ${ }^{1}$ Nephrology \& Hypertension Division, Baruch-Padeh Poriya Medical Center, Lower Galilee, Israel \\ ${ }^{2}$ Department of Pediatrics, Baruch-Padeh Poriya Medical Center, Lower Galilee, Israel \\ ${ }^{3}$ Department of Neonatology, Baruch-Padeh Poriya Medical Center, Lower Galilee, Israel
}

\begin{abstract}
Introduction: Hypertension (HTN), defined as blood pressure (BP) equal to or above $95^{\text {th }}$ percentile for age, height, gender. Hypertension has a prevalence of up to $5-8 \%$ in children. It is a well-known risk factor for cardiovascular diseases affecting the heart, brain and kidneys. HTN presenting at a younger age correlates with increased severity and likelihood of finding an underlying reason for it, therefore defining it as secondary HTN. Recent studies reveal that premature infants $(<37$ weeks) commonly have higher BP, leading to the possibility of a certain connection between the prematurity and hypertension in future.
\end{abstract}

Methods and materials: 87 prematurely born children were examined by criteria of BP measurements at birth and at present in order to evaluate their tendency for HTN in respect to the extent of their prematurity (2009-2018).

Results: Those children presented an increased prevalence of both infantile and childhood HTN (16.7\% and $20.7 \%$ respectively), compared to general population. A negative correlation between birth week and childhood BP values was found, meaning that BP increased monotonically by earlier gestational age, and was found to be statistically significant in diastolic BP.

Conclusions: Prematurely born individuals are at a higher risk to develop childhood HTN. These results encourage the initiation of screening programs for this population.

\section{Introduction}

Hypertension (HTN) has a prevalence of up to5-8\% in children [1]. HTN increases the risk of ischemic heart disease, intracranial hemorrhage and renal disease. Early diagnosis and treatment can helps decrease or even prevent those damaging effects [2-5].

Blood pressure (BP) increases with body mass, those measurements should relate to a percentile according to the gender, age and height of the child [6,7]. Normal BP has systolic \& diastolic values under the $90^{\text {th }}$ percentile, on three measurements. Pre-HTN is defined as BP between 90-95 percentile or over $120 / 80$ up to $95^{\text {th }}$ percentile, while HTN is BP equal to or above $95^{\text {th }}$ percentile for height; gender; age [7].

\section{Etiology}

In adults almost $90 \%$ of HTN is primary, while in children about $80 \%$ is secondary. The difference stems from the adolescence period in which the prevalence of primary HTN increases substantially [4]. Secondary HTN at childhood is related to obesity, chronic conditions, cardiac disease; endocrine disease; renal disease; neurological disease, post-surgical state, drugs birth control pills in adults [6-9]. Prematurely born children or those with a low birth weight have been found to be candidates of pre-HTN [10].

\section{Prematurity and hypertension}

Preterm birth is defined as a birth at fewer than 37 weeks of gestation. Depending on how early an infant is born, he or she may be: late preterm (born between 34 and 36 weeks of gestation), moderately preterm (32-34 weeks), very preterm ( $<32$ weeks) or extremely preterm (born at or before 28 weeks of gestation) [1]. The prevalence of preterm birth in developed countries is about $5-7 \%$, and up to $12 \%$ in the USA [1]. Recent studies reveal that premature infants commonly have higher BP [10-12]. The reason for it remains unclear. Some blamed low birth weight, but no significant difference in BP has been found between infants with low birth weight respective to their gestational age (SGA) and infants with appropriate weight for their gestational age (AGA) [9].

The research of Shankaran, et al. supports this theory by displaying higher BP values among children in term with IUGR compared to a control group [13]. The pathophysiology of this may be related to incomplete intra-uterine growth of the kidney, among other reasons $[14,15]$.

${ }^{\star}$ Correspondence to: Wael Nasser, Nephrology \& Hypertension Division, Baruch-Padeh Poriya Medical Center, Azrieli Faculty of medicine, Israel, Tel: 0506267756; E-mail: wael-nasser@hotmail.com

Key words: gestational age, prematurity, blood pressure, hypertension, cardiovascular diseases

Received: January 07, 2021; Accepted: January 18, 2021; Published: January 21, 2021 


\section{Methods and materials}

\section{Study design}

This research is both of retrospective and prospective, examining the relationship between prematurity and HTN in childhood.

\section{7 constitute the study population}

Children with comorbidities such as any type of syndromes, a single kidney or an ectopic kidney were excluded from the trial.

\section{Data collection}

Demographic data and clinical data regarding the birth was extracted from medical archives. Measurements of BP, weight and height were taken, in addition to blood and urine tests to evaluate kidney function. Statistical analysis

Data analysis was performed using SPSS software, $25^{\text {th }}$ version. Correlation between quantitative variables was assessed using the Spearman Correlation test. Correlation between qualitative variables was assessed using Chi-square test. Correlation between two groups with continuous variables was assessed using T-test. For evaluation of statistical significance, a P-value of 0.05 or under was used.

\section{Results}

\section{Demographic data of research population}

Overall, this research examined 87 pediatric patients born before 37 weeks of gestation. The male to female ratio among our subjects (Table 1) was consisting with the ratio in Israel's northern district [16]. Also the distribution of ethnic origin is consistent to this area, with a slightly higher prevalence of Arab-Muslims in our research population (43.7\% of subjects compared to $37.7 \%$ in general population).

Our research population are 1-10 years old. The mean age is 7.34 with a standard deviation (SD) of 2.08. The distribution of ages according to genders is similar. Observing the birth weight of our subjects, the lowest birth weight recorded was $0.875 \mathrm{~kg}$ and the highest was $3.52 \mathrm{~kg}$. Overall, the mean birth weight is $2.20 \mathrm{~kg}$, with a SD of 0.58 .

The mean gestational age among our subjects is 34.56 (equivalent to 34 weeks and 4 days), with a SD of 2.25. The minimal value is 27.14 (27 weeks and 1 day) and the maximal is 36.86 ( 36 weeks and 6 days). The distribution is presented in (Figure 1).

\section{Hypertension prevalence in research population}

BP measurements on the first day of life were recovered from medical archive for 72 patients. BP measurements were categorized into groups of Normal BP $\left(<90^{\text {th }}\right.$ percentile $)$, Pre-HTN $\left(90^{\text {th }}-95^{\text {th }}\right.$ percentile) and HTN ( $\geq 95^{\text {th }}$ percentile), according to Pejovic, et al.

Table 1. Demographic data of research population

\begin{tabular}{|l|l|l|l|}
\hline \multirow{2}{*}{ Gender } & Count & $\%$ \\
\hline \multirow{2}{*}{ Pregnancy } & Male & 47 & $54 \%$ \\
\hline & Female & 40 & $46 \%$ \\
\hline \multirow{5}{*}{ Ethnicity } & Singleton & 45 & $51.7 \%$ \\
\cline { 2 - 4 } & Multiple gestation & 42 & $48.3 \%$ \\
\hline & Jewish & 30 & $34.5 \%$ \\
\hline & Arab Muslim & 38 & $43.7 \%$ \\
\hline & Arab Christian & 7 & $8 \%$ \\
\hline & Druze & 8 & $9.2 \%$ \\
& Circassian & 1 & $1.1 \%$ \\
\hline & Other & 3 & $3.5 \%$ \\
\hline
\end{tabular}

Studies [17]. Overall, 58 of the subjects $(80.6 \%)$ were normotensive at birth, 2 of them (2.8\%) had Pre-HTN and 12 (16.7\%) had HTN at birth (Figure 2). Current BP was measured from all 87 subjects compiling this study and grouped according to American Academy of Pediatrics' 2017 Clinical Practice Guidelines [18]. Overall, only 53 of the subjects (60.9\%) are currently normotensive, 16 of them (18.4\%) have preHTN and 18 (20.7\%) have HTN. When reviewing the incidence of HTN according to gender, no substantial difference was found (Figure 3). HTN prevalence was also examined in respect to the ethnicities comprising the Galilee region (Figure 4). Among the Arab-Christian population a substantially higher prevalence of Pre-HTN (28.6\%) was noted. Similarly, Druze patients had a higher prevalence of Pre-HTN (12.5\%), Stage 1-HTN (25\%) and Stage 2-HTN (12.5\%). No connection between ethnicity and BP was found to be significant ( $\mathrm{P}$-value $=0.590$, $0.173,0.891$ for total BP, systolic \& diastolic accordingly).

\section{Characteristics of current BP groups}

A statistical analysis was performed on several criteria in an effort to define the main features of each BP group, according to current BP measurements (Table 2). Birth week and birth weight maintained a coordinated mean value in every BP group, suggesting of a strong association between the two. Overall, the mean birth week of normotensive patients and pre-hypertensive ones was similar, at about 34 weeks of gestation. Paradoxically, patients with Stage I HTN had a higher mean of gestational age compared to patients with Stage II HTN. The mean birth weight of those groups followed the same pattern.

\section{Blood pressure values correlation to birth week in prematurely born children}

In order to establish a correlation between birth week and BP values, the Median of birth week (35.14) was used. A significant positive correlation between birth week and BP at birth was found ( $\mathrm{P}$-value $=0$, 0.009, 0.001 for systolic, diastolic and Mean Arterial Pressure (MAP) respectively). Systolic and MAP at birth had a medium strength while diastolic BP showed only a weak correlation. This means that a lower birth week correlates to lower BP values at birth specifically (Table 3). When inspecting the connection between birth week and current BP, we can see a negative correlation of low strength. Hence, a lower birth week correlates to higher BP values. This correlation is significant in diastolic BP measurements ( $\mathrm{P}$-value $=0.036)$, but not in the systolic and $\mathrm{MAP}$ ones ( $\mathrm{P}$-value $=0.473,0.073$ respectively).

Distribution of BP groups according to levels of prematurity (Figure 5) did not detect a specific pattern, though it is suggesting a higher prevalence of HTN in those born 'late preterm' (34-36+6 weeks).

To further explore the effect of prematurity on HTN, we checked whether or not the research population kept their BP percentiles from birth to present days. Of the 58 children who have had a normal BP at birth, 37 (63.8\%) kept a normal BP to today, but the rest, 21 children (36.2\%) progressed to pre-HTN or HTN (10 (17.2\%), 11 (19\%) respectively).

When examining the population with pre-HTN according to current BP measurements, we can see that $71 \%$ of them were normotensive at birth. This is true also for the population with stage I HTN (73\% of them were normotensive at birth) and stage II HTN (all of them were normotensive at birth) (Figure 6).

\section{Factors in the neonatal period influencing current BP}

Different medical conditions and interventions in the neonatal period may explain any variance between $\mathrm{BP}$ values at birth 
Table 2. Characteristics of current blood pressure groups

\begin{tabular}{|c|c|c|c|c|c|c|c|c|c|}
\hline & & \multicolumn{2}{|c|}{ Normal BP } & \multicolumn{2}{|c|}{ Pre-HTN } & \multicolumn{2}{|c|}{ Stage 1 HTN } & \multicolumn{2}{|c|}{ Stage 2 HTN } \\
\hline & & Mean & SD & Mean & SD & Mean & $\mathrm{SD}$ & Mean & SD \\
\hline \multirow{4}{*}{ Total BP } & Birth week & 34.51 & 2.29 & 34.25 & 2.76 & 35.34 & 1.51 & 33.68 & 1.53 \\
\hline & Birth weight & 2.20 & 0.52 & 2.18 & 0.66 & 2.34 & 0.73 & 1.85 & 0.42 \\
\hline & Current age & 7.62 & 1.99 & 6.81 & 2.01 & 7.64 & 1.69 & 4.75 & 3.30 \\
\hline & Current BMI & 16.33 & 2.50 & 16.49 & 2.73 & 15.45 & 2.54 & 15.63 & 3.70 \\
\hline
\end{tabular}

Table 3. Blood pressure values correlation to birth week in prematurely born children

\begin{tabular}{|l|c|c|c|c|c|c|c|}
\hline & & \multicolumn{3}{|c|}{ BP measurement at birth } & \multicolumn{2}{c|}{ Current BP measurement } \\
\hline & & Systolic & Diastolic & MAP & Systolic & Diastolic \\
\hline \multirow{3}{*}{ Week of birth } & Pearson Correlation & 0.436 & 0.281 & 0.379 & -0.007 & -0.194 \\
\cline { 2 - 7 } & P-value (Significant< $\mathbf{0 . 0 5}$ & 0 & 0.009 & 0.001 & 0.946 & 0.036 \\
\cline { 2 - 7 } & Number of subjects & 72 & 72 & 72 & 87 \\
\hline
\end{tabular}

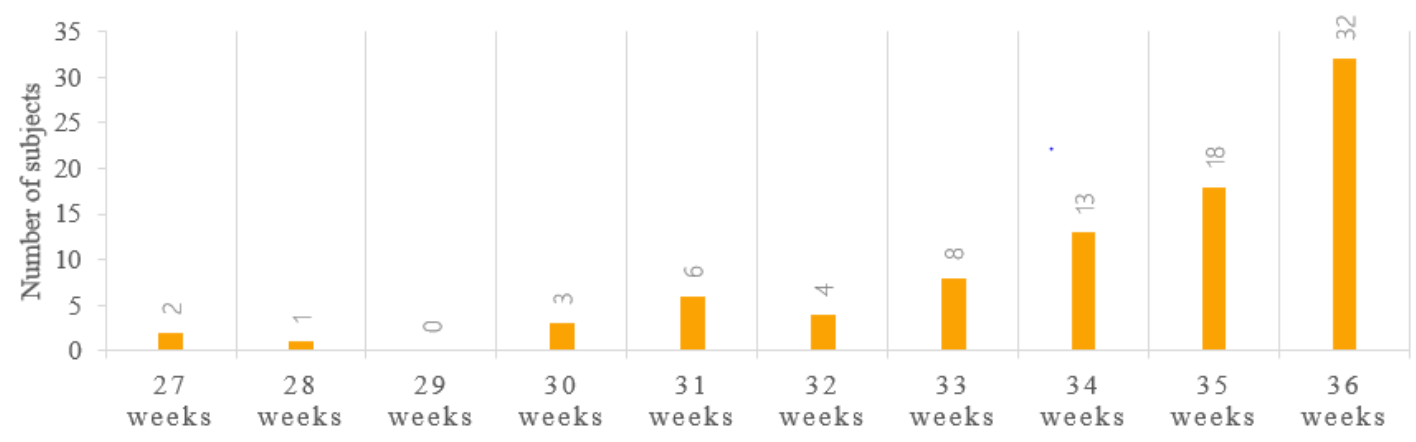

Figure 1. Gestational age distribution

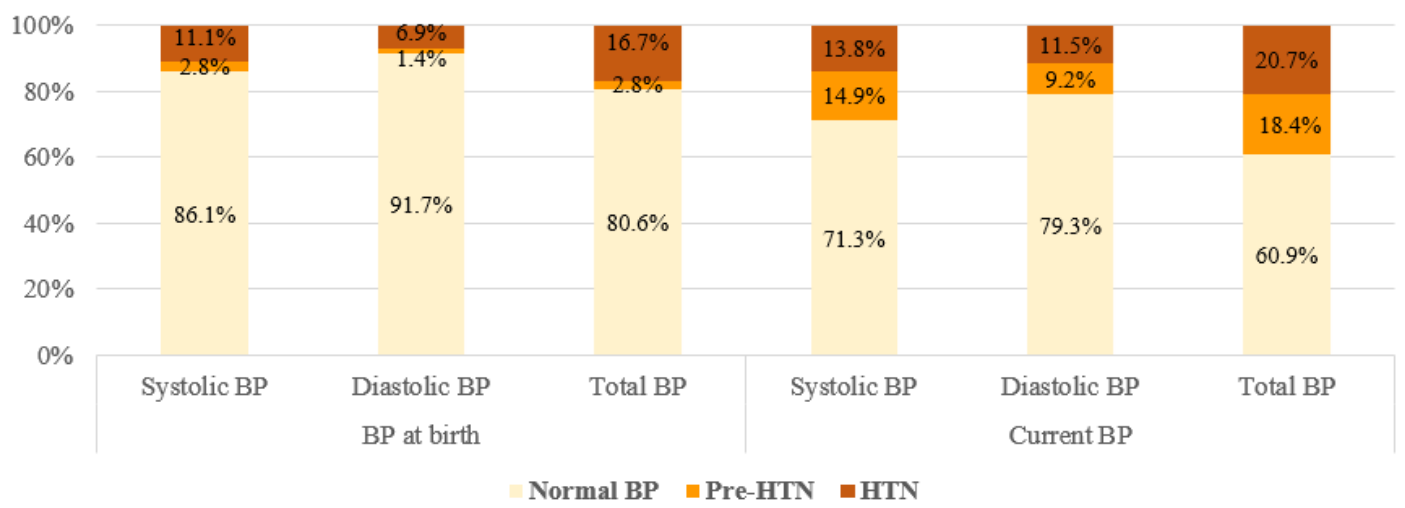

Figure 2. Hypertension prevalence in research population

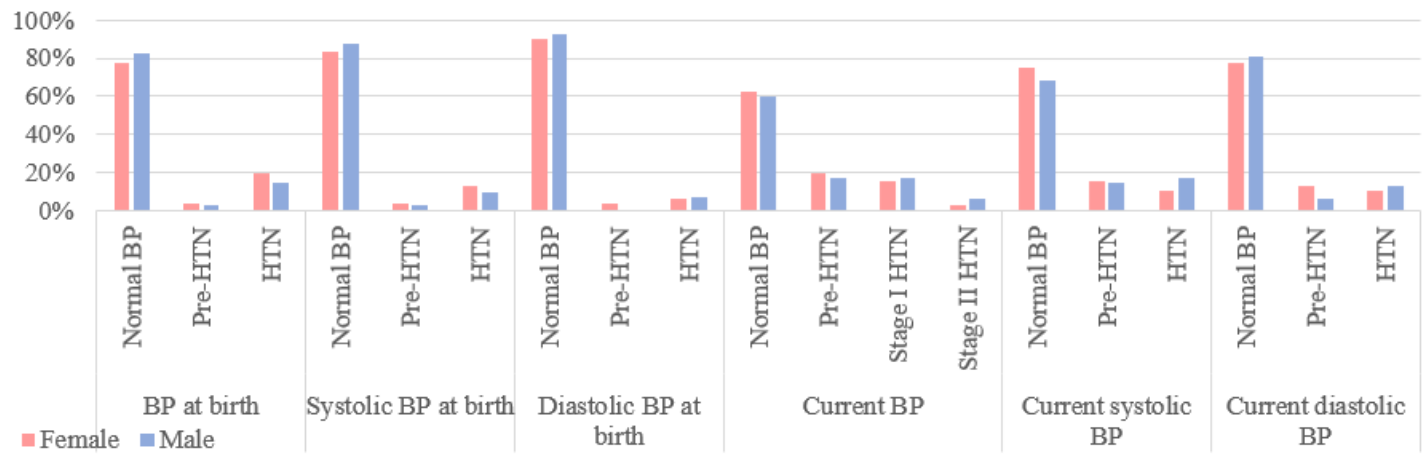

Figure 3. Hypertension prevalence according to gender 


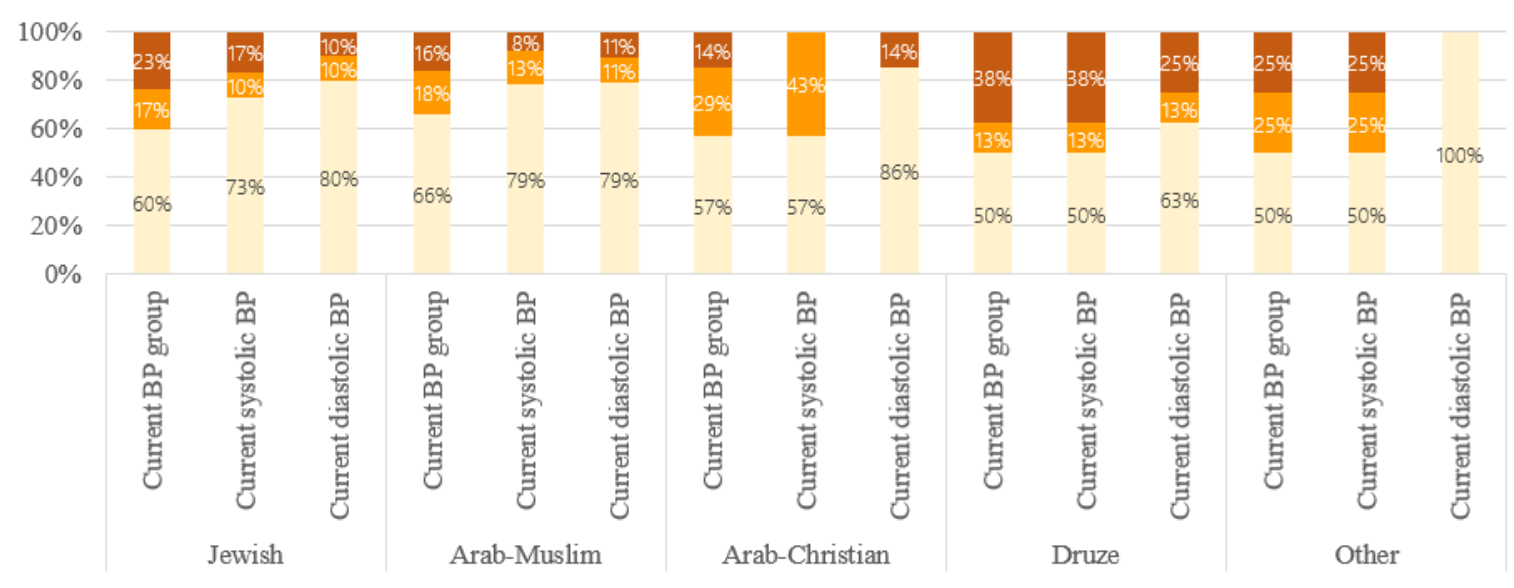

Normal BP $\quad$ Pre-HTN $\quad$ HTN

Figure 4. Hypertension prevalence among different ethnic groups

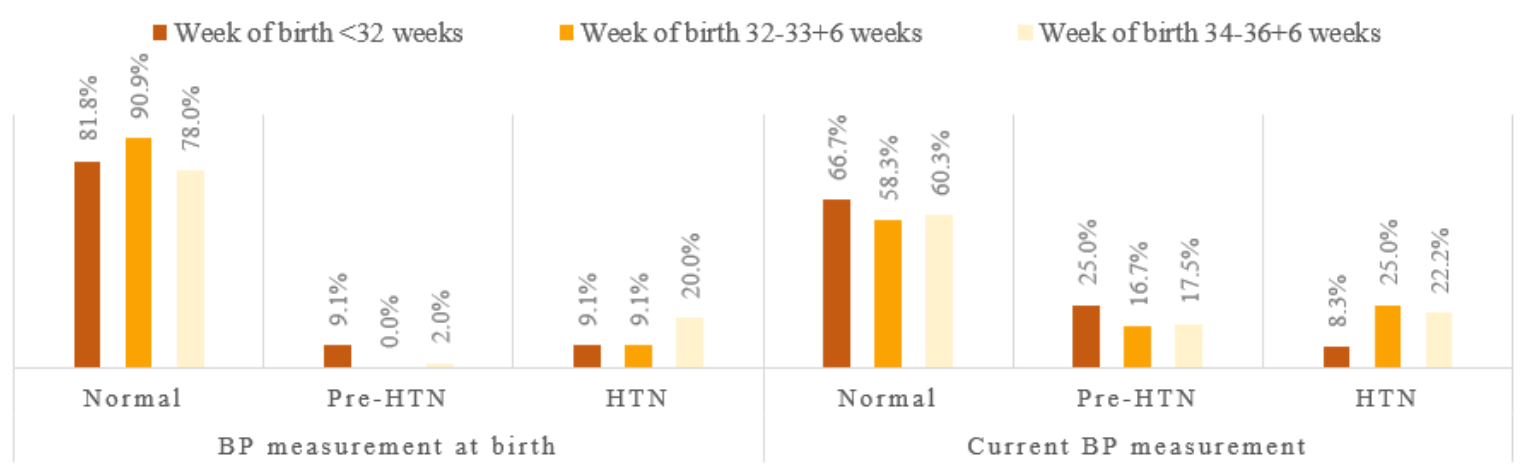

Figure 5. Distribution of BP groups according to gestational age
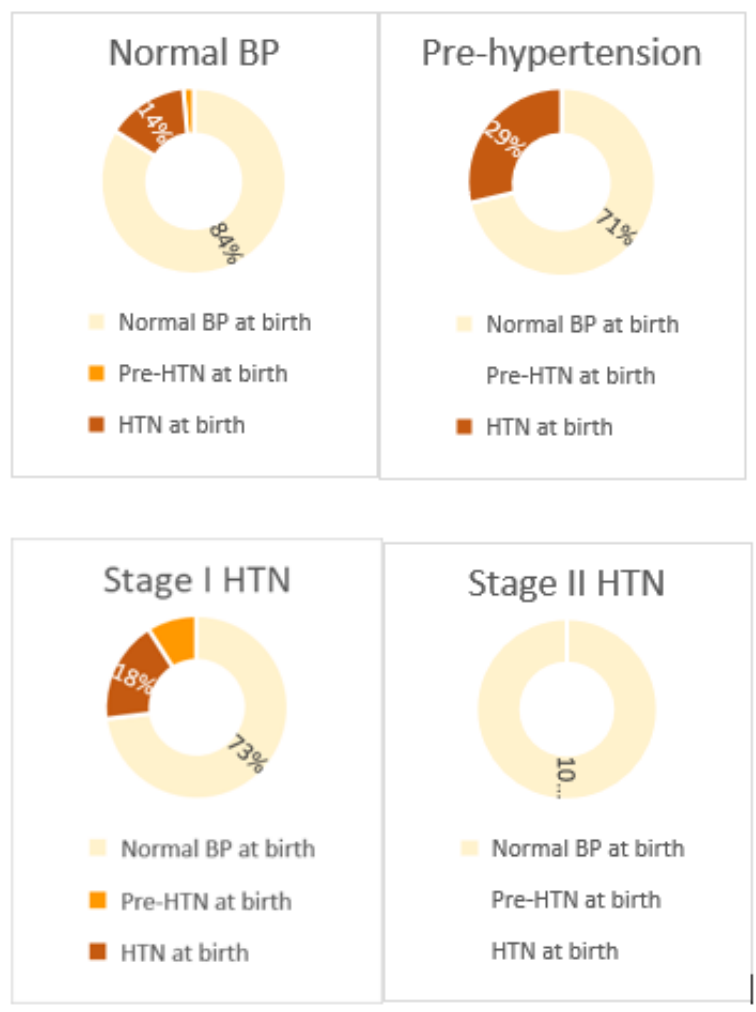

Figure 6. Origin of current blood pressure group and those measured in present days. Patients who underwent umbilical catherization had a higher prevalence of Pre-HTN \& HTN (50\%) compared to others (39.1\%). The correlation between umbilical catherization and current BP is significant in diastolic BP (P-value $=0.013)$. Patients affected with prematurity related conditions investigated show a small increase in Pre-HTN \& HTN states combined (46.2\%) compared to research population (39.1\%). The relationship between BPD and NEC to BP was found to be significant in Diastolic $\mathrm{BP}(\mathrm{P}$-value $=0.0007)$.

\section{Discussion}

In this research we sought to establish a connection between the week of birth and BP values. Our main hypothesis was that prematurity is correlated to a higher risk of HTN in childhood. Though most reports claim that the incidence of HTN in neonates (termed infantile HTN) is quite low, ranging from $0.2 \%-3 \%$ [19] our data showed the incidence among preemies is $16.7 \%$. This can also be seen in current BP measurements. While publications report of a childhood pre-HTN prevalence of $2.2 \%$ to $3.5 \%$ and childhood HTN prevalence of $3.5 \%$ [18], our patients had a prevalence of $18.4 \%$ and $20.7 \%$, respectively. Considering this, one can conclude that prematurity, being the common ground in all of our patients, is correlated to a higher prevalence of elevated BP and HTN.

The prevalence of HTN was found to be similar in males and females. Among the different ethnicities tested, Druze were found to have a particularly high incidence of HTN. This may be explained by a very early gestational age, small sample size and an increased mean 
age among those patients. No updated data regarding the incidence of pediatric HTN in the different ethnicities residing in Northern Israel was found in order to compare.

A certain degree of correlation between the week of birth and BP values was found. At birth, a lower birth week correlated to lower BP values. This result corresponds to Pejovic, et al. and other studies of this nature which determined lower values to be normotensive for premature infants [17]. At childhood we found a negative correlation between the week of birth and current BP values, meaning that an earlier week of birth was associated with higher BP values. This correlation was significant in diastolic BP measurements but not in systolic and MAP measurements. We can assume that these results were insignificant due to the young age of our patients, since similar studies on adolescents are more positive [12], and due to a low portion of children who were born before 32 weeks, which are probably the ones most affected by this condition. In fact, distribution of BP groups according to gestational age suggests a slightly higher prevalence of HTN among those born at $34-36+6$ weeks of gestation. This correlation agrees with the study of Crump, et al. in a Swedish national study of 636,000 births [12]. This study showed that a higher risk of HTN was not only found in correlation to delivery in a very early gestational age, but also among those born near term (35-36 weeks of gestation).

During pregnancy, mothers with gestational diabetes, preeclampsia and medication use had children more prone to childhood HTN. Since both gestational diabetes and maternal use of medication were associated with increased birth week, we can deduce that those conditions are not responsible to the correlation of early birth week and HTN. On the other hand, pre-eclampsia was associated with a lower birth week and with childhood HTN and so might be one of the contributing factors to HTN in this population. This is reasonable considering the fact that maternal HTN is an important risk factor for pre-eclampsia and maternal-child transmission of vascular dysfunction is one of the proposed mechanisms for childhood HTN [12].

Current laboratory measurements of creatinine and urea did not correlate positively to increased BP and therefore do not suggest renal damage as a contributing factor responsible for HTN in this population. It is possible that imaging studies of kidneys may shine another light on this situation. Instead, blood and urine sodium levels showed a very significant positive correlation to BP, indicating some role in HTN development.

Patients diagnosed with HTN in our research had a lower BMI compared to their companions, ergo ruling out obesity, a major risk factor for HTN, as the reason for the increased HTN prevalence found among our patients [18].

\section{Conclusion}

To summarize, this study suggests that prematurity correlates to lower BP at birth, but also to higher BP in childhood. In addition, prematurely born children have a much higher prevalence of elevated $\mathrm{BP}$ compared to general population, even in those born near term.
These results encourage the initiation of HTN screening programs for this population. This work can aid in early diagnosis and treatment of such individuals, which are crucial in order to delay and possibly prevent the development of HTN-related morbidity and mortality in adulthood.

\section{References}

1. Tucker J, McGuire W (2004) Epidemiology of preterm birth. BMJ 329: 675-678. [Crossref]

2. Martin JA, Kung HC, Mathews TJ, Hoyert DL, Strobino DM, et al. Annual summary of vital statistics: 2006. Pediatrics 121: 788-801.

3. Lahra MM, Jeffery HE (2004) A fetal response to chorioamnionitis is associated with early survival after preterm birth. Am J Obstet Gynecol 190: 147-151. [Crossref]

4. Claas MJ, de Vries LS, Bruinse HW, van Haastert IC, Uniken Venema MM, et al (2011) Neurodevelopmental outcome over time of preterm born children $\leq 750 \mathrm{~g}$ at birth. Early Hum Dev 87: 183-191. [Crossref]

5. Kwinta P, Pietrzyk JJ (2010) Preterm birth and respiratory disease in later life. Expert Rev Respir Med 4: 593-604.

6. Bacchetta J, Harambat J, Dubourg L, Guy B, Liutkus A, et al. (2009) Both extrauterine and intrauterine growth restriction impair renal function in children born very preterm. Kidney Int 76: 445-452.

7. Keijzer-Veen MG, Finken MJJ, Nauta J, Dekker FW, Hille ETM, et al. (2005) Is blood pressure increased 19 years after intrauterine growth restriction and preterm birth? A prospective follow-up study in the Netherlands. Pediatrics 116: 725-731. [Crossref]

8. Kwinta P, Pietrzyk JJ (2007) Retinopathy of prematurity: is genetic predisposition an important risk factor?. Expert Rev Ophthalmol 2: 275-283.

9. Rodríguez-Soriano J, Aguirre M, Oliveros R, Vallo A (2005) Long-term renal followup of extremely low birth weight infants. Pediatr Nephrol 20: 579-584.

10. Bayrakci US, Schaefer F, Duzova A, Yigit S, Bakkaloglu A (2007) Abnormal circadian blood pressure regulation in children born preterm. J Pediatr 151: 399-403.

11. Keijzer-Veen MG, Dülger A, Dekker FW, Nauta J, Van Der Heijden BJ (2010) Very preterm birth is a risk factor for increased systolic blood pressure at a young adult age. Pediatr Nephrol 25: 509-516. [Crossref]

12. Crump C, Winkleby MA, Sundquist K, Sundquist J (2011) Risk of hypertension among young adults who were born preterm: a Swedish national study of 636,000 births. Am J Epidemiol 173: 797-803. [Crossref]

13. Shankaran S, Das A, Bauer CR, Bada H, Lester B, et al. (2006) Fetal origin of childhood disease: intrauterine growth restriction in term infants and risk for hypertension at 6 years of age. Arch Pediatr Adolesc Med 160: 977-981.

14. McElrath TF, Hecht JL, Dammann O, Boggess K, Onderdonk A, et al. (2008) Pregnancy disorders that lead to delivery before the 28th week of gestation: an epidemiologic approach to classification. Am J Epidemiol 168: 980-989.

15. Hovi P, Andersson S, Räikkönen K, Heinonen K, Pyhälä R, et al. (2010) Ambulatory blood pressure in young adults with very low birth weight. J Pediatr 156: 54-59.

16. https://www.cbs.gov.il/he/Settlements/Pages/Localities/Yishuv.aspx?semel=2\&mode=Machoz

17. Pejovic B, Peco-Antic A, Marinkovic-Eric J (2007) Blood pressure in non-critically ill preterm and full-term neonates. Pediatr Nephrol 22: 249-257.

18. Flynn JT, Kaelber DC, Baker-Smith CM, Blowey D, Carroll AE, et al. (2017) Clinical practice guideline for screening and management of high blood pressure in children and adolescents. Pediatrics 140: e20171904. [Crossref]

19. Dionne JM, Abitbol CL, Flynn JT (2012) Hypertension in infancy: diagnosis, management, and outcome. Pediatr Nephrol 27: 17-32.

Copyright: (C2021 Elkayam A. This is an open-access article distributed under the terms of the Creative Commons Attribution License, which permits unrestricted use, distribution, and reproduction in any medium, provided the original author and source are credited. 